\title{
Andropause ou P.A.D.A.M.
}

\author{
R. Schoysman \\ SIMAF, Hôpital Van Helmont, Vilvoorde, Belgique
}

\section{RESUME}

Récemment le vocable P.A.D.A.M. "Partial Androgen Deficiency in Aging Men" a été proposé pour remplacer le terme Andropause. Le terme P.A.D.A.M. implique que la majorité des problèmes de l'homme âgé serait due à une déficience en testostérone. Cela ne se vérifie par par de grandes séries d'examens endocrinologiques. De nombreux autres problèmes se posent à l'homme âgé, auxquels la testotérone n'apporte pas de solution. Le vocable P.A.D.A.M paraît dès lors peu approprié.

Mot-clés: andropause, testostérone, sexualité

\section{INTRODUCTION}

Le mot "Andropause" a été introduit dans le vocabulaire médical pour désigner chez l'homme un certain nombre d'altérations fonctionnelles au fur et à mesure qu'il avance en âge. Ce vocable prend donc le contre-pied du terme "ménopause" qui comprend des situations comparables quoique non similaires chez la femme.

Le terme P.A.D.A.M. est l'abréviation de Partial Androgen Deficiency in Aging Men. Ce vocable restreint très fortement celui qui est défini par le terme "andropause" puisque tout revient à accuser une diminution de la testostéronémie d'être la cause de tous les problèmes de l'homme âgé.

Si le terme P.A.D.A.M est de fabrication récente et si le terme ménopause existe depuis des siècles, le mot andropause est entré dans le vocabulaire médical, voire même populaire, depuis deux générations seulement. La cause en est un très important accroissement de la durée de vie, puisqu'au début du siècle la longévité moyenne de l'homme était de 50 , alors qu'actuellement elle atteint 75 ans. Il s'ensuit que l'étendue des problèmes de l'homme âgé touche à ce jour un nombre plus important de mâles, et que les médecins, les psychologues, les sexologues et les sociologues sont confrontés avec une pathologie beaucoup plus fréquente qu'il y a deux générations [5].

La différence fondamentale entre les concepts ménopause et andropause réside dans le caractère brusque de l'apparition de la ménopause qui peut s'installer en quelques mois ou au maximum en quelques années. Le symptôme le plus marquant est l'arrêt des menstruations, de façon brusque ou progressive et précédé de longues périodes d'oligospanioménorrhée. Chez l'homme, l'andropause s'installe d'une façon beaucoup plus lente et insidieuse. [4].

Pour l'installation de l'andropause, on retrouve, comme pour toutes les autres fonctions ou particularités anatomiques de l'homme, une distribution gaussienne sur laquelle se projettent non seulement des caractéristiques physiques, mais aussi l'activité intellectuelle, sexuelle et également la fertilité. En effet, certains hommes restent intellectuellement très créatifs jusqu'à un âge très avancé et dans le domaine de la sexualité et de la fertilité, il en est de même puisqu'il existe des dossiers bien étudiés d'hommes qui ont encore fécondé à l'âge de 90 ans. 


\section{LES MODIFICATIONS PHYSIQUES}

Parmi les modifications physiques, on note:

Une reprise plus lente et plus tardive après une maladie ou un traumatisme.

Une résistance plus brève aux activités physiques.

Une tendance à l'excès pondéral.

Une difficulté à lire des caractères d'imprimerie plus petits.

Une progressive chute de cheveux.

Des recherches de densité osseuse montrent qu'une ostéoporose subclinique s'installe, tout comme chez la femme mais avec un retard de plus de 10 ans par rapport à celle-ci [7-8].

Toutes ces modifications peuvent être - du moins en partie - attribuées à une décroissance de l'androgénicité dont le substrat biochimique est un déclin de la sécrétion de testostérone (fig. 1) [9]. Dans la fonction testiculaire, ce n'est pas que la cellule de Leydig qui connaît une diminution fonctionnelle liée à l'âge (2), mais également la cellule de Sertoli, clé de la spermatogenèse. Les effets bénéfiques signalés par la compensation en androgènes soulignent l'importance de cette testostéronémie. En gros, on peut affirmer qu'à l'âge de 80 ans, la testostéronémie n'est plus qu'à $60 \%$ de la valeur observée chez l'homme de 25 ans. Cela va logiquement de pair avec une élévation de la F.S.H. (fig. 2) [2-7]. Il a aussi été démontré que la testostérone biologiquement active débute sa courbe descendante vers l'âge de 35 ans.

\section{MODIFICATIONS PSYCHOLOGIQUES}

L'homme qui avance en âge est plus facilement sujet à des phases d'irritabilité, d'anxiété, de peur et de dépression. Il peut perdre confiance en soi, éprouver de plus en plus de difficultés à être joyeux et connaître une sensation de solitude, de ne plus être attrayant et de ne plus être aimé.

Il serait cependant erroné de considérer ces modifications psychologiques comme étant liées à une diminution de la testostérone. En effet, des facteurs sociologiques jouent un rôle

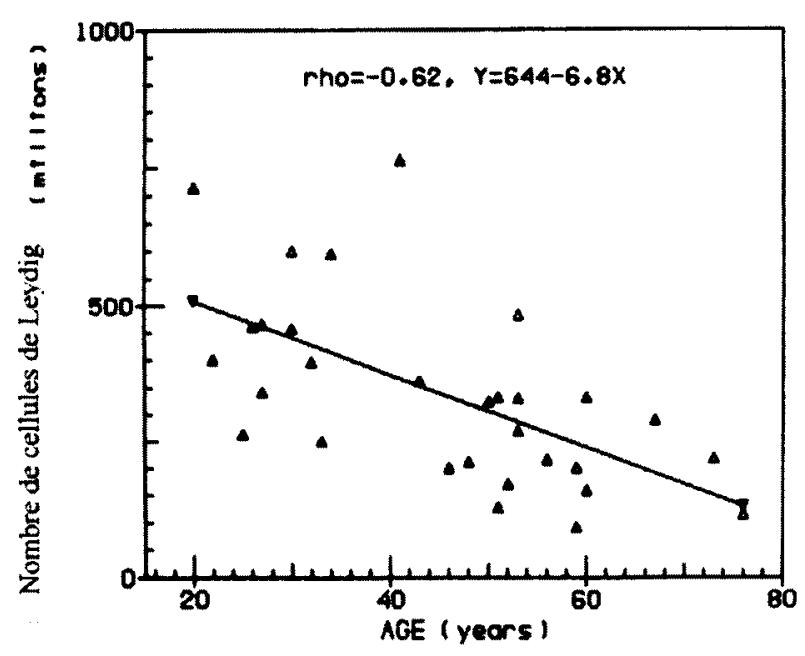

Figure 1 : Le déclin de la fonction leydigienne est progressif et débute vers la quarantaine [8].

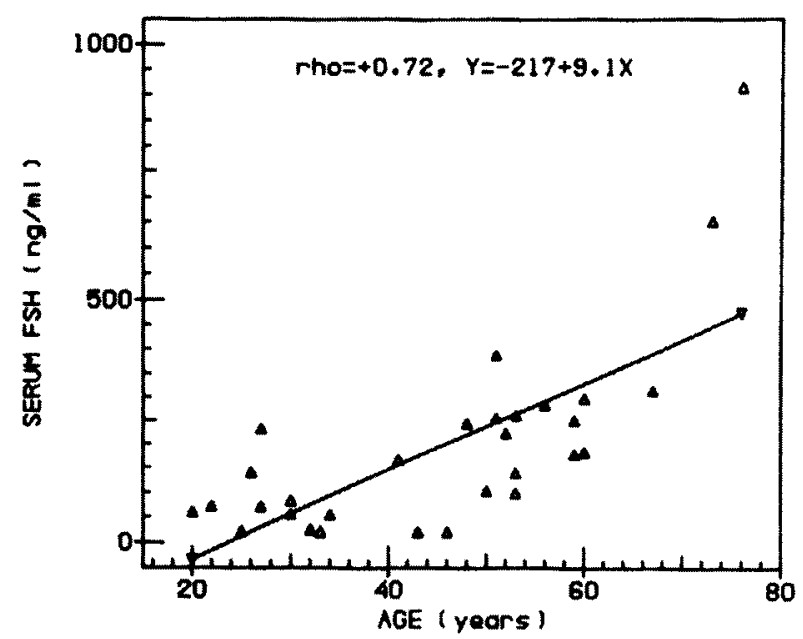

Figure 2 : Evolution des taux de FSH en fonction de l'âge [8].

prépondérant dans la psychologie de l'homme âgé. Le développement fulgurant de la technicité dans le travail est pour beaucoup une évolution de plus en plus difficile à suivre. Ainsi, par exemple, l'homme plus jeune captera beaucoup plus rapidement toutes les astuces de l'ordinateur parce que ce dernier faisait déjà partie de son éducation et le fera de plus en plus. Le marché du travail est tel que l'homme de 40 ans qui "perd son emploi" a des difficultés considérables pour en retrouver un autre. Il n'est dès lors nullement étonnant qu'un manque de confiance en soi s'installe, parce 
qu'il est à ce jour un fait de société d'être mis à l'écart dès qu'on n'est plus à la pointe de la créativité. L'homme de 50 ans ou plus, qui a toujours une famille dont il doit s'occuper, se trouve dès lors dans une situation très angoissante et stressante.

\section{LA DIMINUTION DE LA FERTILITÉ}

Le désir de procréer de l'homme âgé est un phénomène sociologique neuf. On voit à l'heure actuelle beaucoup plus fréquemment dans les consultations d'andrologie, des hommes ayant largement dépassé la soixantaine et qui reviennent consulter avec une plus jeune épouse dans le désir d'avoir un enfant. Des travaux très précis ont montré que dans le testicule il y a une diminution de la quantité de cellules de Leydig et des cellules de Sertoli. Les répercussions sur la spermatogenèse ont été bien mises en évidence [3]. Une nouvelle fois cette situation est à projeter sur une courbe gaussienne. L'homme qui acquiert à la puberté des gonades plus riches en éléments Sertoliens ou Leydigiens ne connaîtra que beaucoup plus tardivement des problèmes de diminution de fertilité.

Le sperme se ressent bien entendu de cette diminution de la fonction testiculaire et les valeurs moyennes des numérations des spermatozoïdes dans les éjaculats le soulignent (fig.3). Cependant, même chez des patients très âgés, mais dans de bonnes conditions de santé générale, la diminution de la spermatogenèse ne va pas compromettre de façon significative la fertilité du sujet. Il y a globalement une diminution de $25 \%$ de la qualité des paramètres biologiques du sperme, et dès lors du pouvoir fécondant chez les hommes âgés par rapport aux plus jeunes.

\section{L'ACTIVITÉ SEXUELLE}

L'activité sexuelle est nettement diminuée chez de nombreux hommes âgés. Leur intérêt pour l'acte sexuel diminue et ils connaissent souvent une anxiété croissante de perdre leur puissance sexuelle. Pour contrebalancer cette anxiété, les fantasmes sont de plus en plus fréquemment invoqués. Beaucoup plus fréquemment que pour l'homme jeune surgissent des

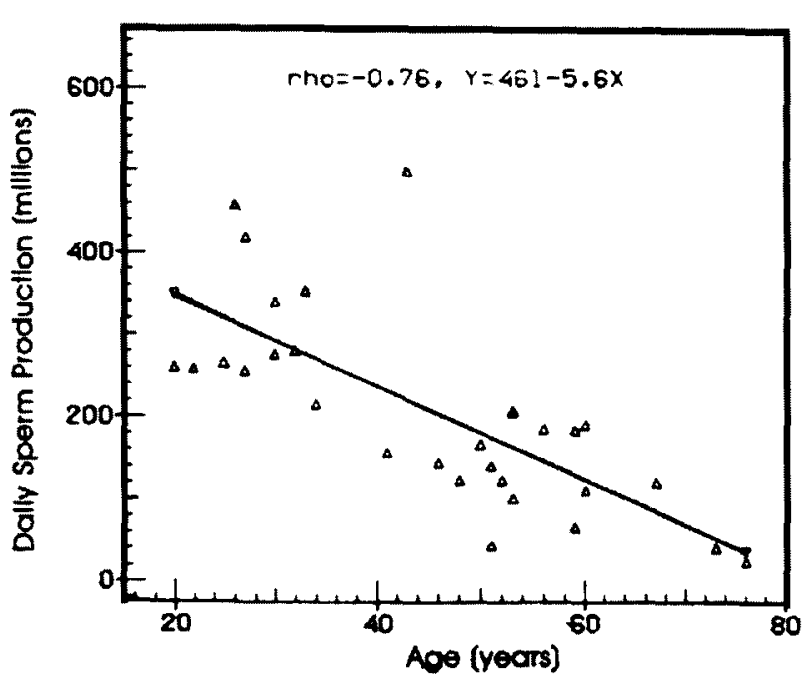

Figure 3 : Production quotidienne de spermatozoïdes en fonction de l'âge [8]

problèmes conflictuels dans leur relations sociales, sexuelles, intimes et même affectives. L'érection demande plus de temps pour se compléter et nécessite des stimuli plus directs. Le besoin impératif d'éjaculer diminue au point de ne plus être pour certains un aboutissement automatique de l'acte sexuel. Le volume et la force de l'éjaculation diminuent. L'examen physique montre une diminution du volume et de la consistance des testicules.

Tout ce tableau ne résulte pas exclusivement d'un abaissement de la testostéronémie. De nombreuses observations prouvent que des perturbations sexuelles importantes sont d'avantage des conséquences de maladies que $\mathrm{du}$ vieillissement. De plus, il faut retenir la longue liste de médications psychotropes ou cardio-vasculaires qui retentissent sur la fonction sexuelle et dont la consommation s'accroît avec l'âge [3].

De nombreuses hormones ont été étudiées pour cerner l'ensemble de ces difficultés. La littérature mentionne ainsi des travaux sur la dopamine, l'ocytocine, la vasopressine, les hormones de croissance, la mélatonine, le DHEA et bien entendu la testostérone.

C'est cette dernière qui est souvent retenue comme l'hormone clé d'une activité sexuelle diminuée; d'où la création du concept P.A.D.A.M. Il est dès lors normal qu'on ait 
tenté chez de tels patients de conseiller l'administration de testostérone par voie intramusculaire, menant à une sensation de bien-être avec une vigueur et une énergie accrue, non seulement psychologiquement mais également par l'augmentation de la masse et de la force musculaires. De nombreux travaux soulignent l'augmentation des globules rouges, de l'hémoglobine et de l'amélioration de la densité osseuse. Il y a de très nombreux arguments contre cette façon de voir : chez seulement $20 \%$ des hommes de 65 ans la testostéronémie descend à des niveaux hypogonadiques. Si certains hommes bénéficient de l'administration de testostérone, il ne faut pas perdre de vue que tous les hommes âgés n'ont pas pour autant une testostéronémie déficiente. Une supplémentation ne devrait être considérée que si le taux des androgènes est dans les valeurs hypogonadiques.

Théoriquement, l'administration d'androgènes de façon continue n'est pas sans risque. Un de ces risques est de stimuler la progression d'un carcinome de la prostate. Le carcinome prostatique subclinique n'est pas décelable et il n'y a pas de marqueur fiable. Il n'y pas d'arguments formels pour affirmer que les androgènes peuvent accélérer la transformation d'un carcinome subclinique et quiescent en une lésion évolutive, mais ce risque n'est pas à exclure.

Il s'ensuit que toute administration de testostérone doit être précédée d'un examen prostatique méticuleux et régulièrement répété. $\mathrm{Si}$ risque il y a, il est largement compensé par les bénéfices que signalent les hommes hypogonadiques.

Un certain nombre d'endocrinologues considèrent l'administration de testostérone comme erronée puisque cette administration exogène ira à l'encontre de la production physiologique. Aussi, l'administration d'androgènes à l'homme âgé connaît à l'heure actuelle les mêmes doutes qui foisonnaient dans la littérature lorsque il a été suggéré pour la première fois qu'il était favorable de donner des oestrogènes à la femme ménopausée et ce pour de très longues périodes.

La diminution relative de la testostéronémie est-elle la seule cause de la diminution de l'ac- tivité sexuelle? Certainement pas. Les modifications psychologiques résultant de facteurs sociologiques, signalés plus haut, ne jouent pas en faveur de la sexualité. Une sexualité heureuse demande un climat d'insouciance que l'homme jeune peut se recréer plus facilement que l'homme âgé. En termes triviaux, l'homme jeune peut laisser les soucis ou les dossiers difficiles sur la descente de lit alors que l'homme âgé les prend avec lui sur l'oreiller.

Quant à l'activité sexuelle, il est très difficile d'en définir la norme. Pour la population ouesteuropéenne, un ou deux rapports par semaine semblent la moyenne avec bien entendu de larges variations en période de vacances et d'insouciance relative. Il y a de nombreuses exceptions à cette affirmation et il faut à nouveau retourner à la courbe de Gauss.

\section{CONCLUSION}

Le phénomène andropause demande donc une approche multidisciplinaire et déborde largement la seule déficience en testostérone. Il est à notre sens erroné de vouloir substituer au vocable andropause, le vocable P.A.D.A.M. qui restreint, et de beaucoup, l'ensemble des problèmes qui se posent à l'homme âgé. Il n'existe pas de données statistiques valables dans ce domaine puisque des hommes sans problèmes ne consultent pas et que sans nul doute beaucoup d'autres s'en accommodent sans voir la nécessité d'un traitement médical. On est en droit de se demander si le rejet du vocable andropause ne trahit pas un sentiment macho qui fait partie du bagage psychologique de nombreux mâles.

Il ne faut pas perdre de vue qu'à l'heure actuelle toute la civilisation du monde occidental va dans un sens de rajeunissement qui ne touche pas seulement la mode vestimentaire mais également le climat psychologique. Le sexe a pris dans le monde du spectacle, dans la littérature et dans toute notre société une place de plus en plus importante. Une propagande commerciale continue faisant largement usage de l'attrait de la beauté plastique, pousse à de nouvelles habitudes non seulement vestimentaires mais aussi relationnelles et sexuelles. Le rejet de l'âge est devenu la préoccupation 
majeure de la vie sociale des individus. Avec pour bon nombre d'entre eux l'oubli d'autres possibilités de richesse d'existence en amitiés ou en amour, non exclusivement focalisés sur l'acte sexuel.

Il reste sous l'angle de la procréation, une conséquence préoccupante à savoir que si la paternité reste possible jusqu'à 60 ans et plus, les risques inhérents au vieillissement des spermatogonies augmentent [1].

Si nos sociétés connaissent actuellement un nombre croissant d'enfants conçus à partir de pères plus âgés, leurs évaluations epidémiologiques futures nous éclaireront d'avantage sur d'éventuelles conséquences de l'actuel vieillissement de nos populations et leur recherche faustienne de l'éternelle jeunesse.

\section{REFERENCES}

1. AUROUX M., Evaluation de la fertilité en fonction de l'âge et risque pour la progénéture, Contracpt. Fertil. Steril., 1991, 19, 11, 945-949.

2. BREMNER WJ, Vitiello MV, Prinz PN Loss of circadian rhythmicity in blood testosterone levels with aging in normal men. J Clin Endocrinol Metab, 1983; 56:1278.

3. DEAMER RL. THOMPSON JF: The role of medications in geriatric sexual function. Clin Geriatr Med, 1991; 7:95.

4. HOLSTEIN A. F., Spermatogenese im Alter - ein Grenzgebiet zwischen normaler und pathologischer Anatomie. Urologie A, 1986; 130-137.

5. JOHNSON Larry, Spermatogenesis and Aging in the Human. J. Clin. Endocrinology Metab., 1987; 331355.

6. JOHNSON L., HUNG BA NGUYEN, PETTY C.S. and NEAVES W.B. : Quantification of human spermatogenesis: germ cell degeneration during spermatocytogenesis and meiosis in testes from younger and older adult men. Bio. Reprod. 37, 1987; 739-747.

7. KALER LW, NEAVES WB Attrition of the human Leydig cell population with advancing age. Anat Rec, 1978, 192:513

8. MEIER DE et al: Marked decline in trabecular bone mineral content in healthy men with age: Lack of association with sex steroid levels. J Am Geriatr Soc, 1987; 35:189.
9. NEAVES W. B., JOHNSON L., PORTER J.C., PARKER C. R. JR., and PETTY C.S., Leydig cell numbers, daily sperm production, and serum gonadotropin levels in aging men. J. Clin. Endocrinology Metab. 1984; 756-761

\author{
ABSTRACT \\ Andropause or P.A.D.A.M. ? \\ R. SCHOYSMAN
}

Recently the word P.A.D.A.M. has been proposed to replace the term Andropause (Partiel Andrologen Deficiency in Aging Males). This implies that most if not all problems of the aging male are due to testosterone déficiency, which is not at all true when one considers the results of endocrine investigations in that group. So many other sociological and relational causes are involved that the term P.A.D.A.M. is clearly a misnomer.

Key-Words : Andropause, testosterone sexuality 